
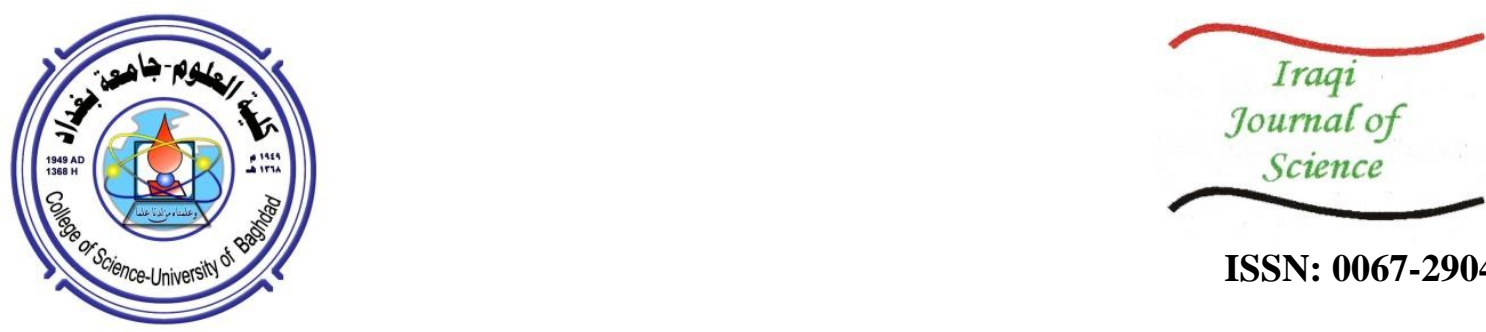

ISSN: 0067-2904

\title{
Chaos in a Hybrid Food Chain Model
}

\author{
Safaa Jawad Ali ${ }^{*}$, Abed Almohsen Naji Almohasin' ${ }^{2}$, Adwea Naji Atewi ${ }^{1}$, Raid Kamel \\ Naji $^{3}$, Norihan Md Arifin ${ }^{4}$ \\ ${ }^{1}$ Institute of Medical Technology/Al-Mansour, Middle Technical University, Baghdad, Iraq, \\ ${ }^{2}$ Middle Technical University, Main University Office, Baghdad, Iraq, \\ ${ }^{3}$ Department of Mathematics, College of Science, University of Baghdad, Baghdad, Iraq, \\ ${ }^{4}$ Institute for Mathematical Research, University Putra Malaysia, Malaysia,
}

Received: $15 / 9 / 2020$

Accepted: 21/12/2020

\begin{abstract}
In this paper, chaotic and periodic dynamics in a hybrid food chain system with Holling type IV and Lotka-Volterra responses are discussed. The system is observed to be dissipative. The global stability of the equilibrium points is analyzed using Routh-Hurwitz criterion and Lyapunov direct method. Chaos phenomena is characterized by attractors and bifurcation diagram. The effect of the controlling parameter of the model is investigated theoretically and numerically.
\end{abstract}

Keywords Bifurcation diagram, Chaos, Global stability, Holling type IV, LotkaVolterra.

\section{الفوضى في نموذج السلسلة الغذائية الهجينة}
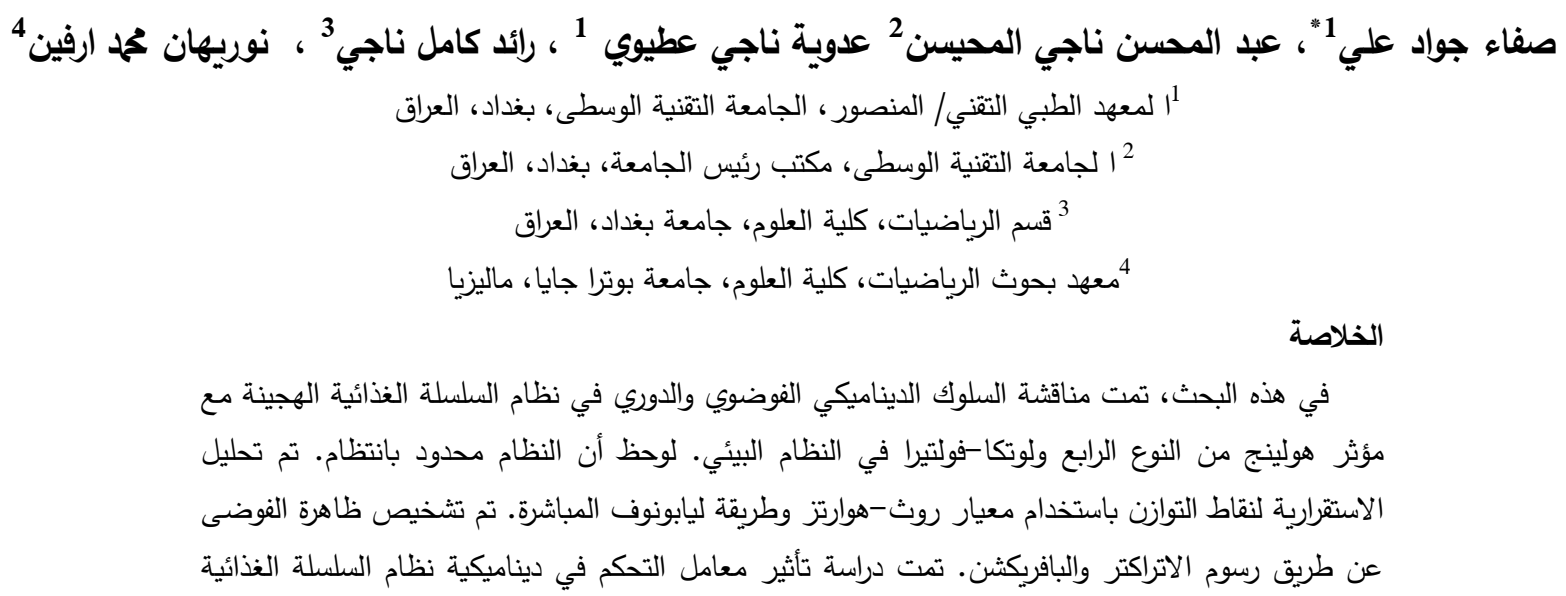$$
\text { تحليليا وعدديا. }
$$

\section{Introduction}

Nonlinear phenomena play important rules in design systems of engineering and structures. The works of Lotka [1] and Volterra [2] are the most major works in mathematical models and dynamical systems. Researchers in ecology and engineering reported the essence of chaos [3-12]. In the analysis of the mechanical model, the geometrical non-linearity, represented by the pendulum, may lead to chaotic dynamics $[4,13]$. Continuous three species food chain models from dissipative dynamics are accounted for to have complicated patterns, such chaos limit cycle, and stable cycle [14-17]. Two 
types of hybrid functional responses of a food chain model were investigated [18] and the numerical works proved the existence of chaotic dynamics. Upadhyay et al. [19] proposed and studied a tritrophic food chain model with Sokol-Howell functional response, incorporating the multiple gestation time delays. They explored the existence of various dynamical structures, such as Hopf-bifurcation, periodic solutions, and chaotic dynamics. Fayeldi et al. [20] applied Euler method to discretize a SIR epidemic model with non-monotone incidence rate. They showed analytically and numerically that the discrete model is dynamically consistent with its continuous model. Liu and Huang [21] investigated the dynamical behavior of a predator-prey system with Holling type IV functional response, in which both species are subject to harvesting. They illustrated that using the harvesting effort as a control parameter can change the behaviors of the system, which may be useful for the biological management.

The primary motivation behind this work is to examine the dynamics of a three species model. A food chain model with Lotka-Volterra and Holling type IV functional responses is proposed and studied both theoretically and numerically. The food chain model is modified to the food chain model studied in [9], so that it involves Lotka-Volterra type of functional response in the second and third levels, instead of Holling type-II functional response that was used in the previous work. The idea of such modification comes from the fact of availability of food at the second level in the environment. The second reason for such modification is that we want to reduce the intensity of nonlinearity in the system and then study the effects of such reduction on the existence of chaos.

\section{The Mathematical Model}

Consider a three species food chain model consisting of the prey, their density at time $t$ is denoted by $x(t)$, the intermediate predator, their density at time $t$ is denoted by $y(t)$, and the top predator, their density at time $t$ is denoted by $z(t)$. It is assumed that the intermediate predator preys upon its sole food at the lower level according to a modified Holling type IV functional response, and the top predator preys upon the intermediate predator at the second level according to Lotka-Volterra functional response. The dynamics of the above food chain model can be represented by the following system:

$$
\begin{aligned}
& \frac{d x}{d t}=a x-b x^{2}-\frac{w_{1} x y}{h+x^{2}}=F_{1}(x, y, z) \\
& \frac{d y}{d t}=\frac{w_{2} x y}{h+x^{2}}-d_{1} y-w_{3} y z=F_{2}(x, y, z) \\
& \frac{d z}{d t}=w_{4} y z-d_{2} z=F_{3}(x, y, z),
\end{aligned}
$$

with $x(0) \geq 0, y(0) \geq 0, z(0) \geq 0$. Obviously, system (1) is continuous and has continuous partial derivatives on the positive octant $R_{+}^{3}=\{(x, y, z) \in \mathrm{x}(0) \geq 0, \mathrm{y}(0) \geq 0, \mathrm{z}(0) \geq 0\}$, and hence the solution of the system (1) exists and is unique.

Here, the positive constants $a, b, d_{j}, h ; j=1,2$ and $w_{k} ; k=1,2,3,4$ denote the following: $a$ is the growth rate of the prey $x, b$ represents the intraspecific competition of prey $x, w_{k}$ represent the maximum values attainable by each per capita rate, $h$ is the half-saturation constant, and $d_{j}$ represent the death rates of the intermediate and the top predators, respectively. Moreover, it is easy to verify that system (1) is uniformly bounded.

\section{Stability Analysis}

This section deals with the stability analysis of the non-negative equilibrium points of system (1). There are at most four non-negative equilibrium points, which are denoted respectively as $E_{0}, E_{1}, E_{2}$ and the interior equilibrium point $E_{3}$.

The trivial equilibrium point $E_{0}=(0,0,0)$ and the axial equilibrium point $E_{1}=\left(\frac{a}{b}, 0,0\right)$ always exists. While the planer equilibrium point is $E_{2}=(\bar{x}, \bar{y}, 0)$ where

$$
\bar{x}=\frac{w_{2}}{2 d_{1}} \mp \frac{\sqrt{w_{2}{ }^{2}-4 d_{1}{ }^{2} h}}{2 d_{1}} ; \bar{y}=\frac{(a-b \bar{x})\left(h+\bar{x}^{2}\right)}{w_{1}} .
$$

Clearly, there are two positive planar equilibrium points denoted by $E_{21}=\left(\bar{x}_{1}, \bar{y}_{1}, 0\right)$ and $E_{22}=$ $\left(\bar{x}_{2}, \bar{y}_{2}, 0\right)$ if and only if the following condition holds

$$
w_{2}^{2}-4 d_{1}^{2} h>0 \text {, }
$$


while system (1) has a unique planar equilibrium point $E_{2}=(\bar{x}, \bar{y}, 0)$ provided that

$$
w_{2}{ }^{2}=4 d_{1}{ }^{2} h \text {. }
$$

The positive equilibrium point $E_{3}=\left(x^{*}, y^{*}, z^{*}\right)$ exists in the interior of the first octant if and only if the following conditions hold

$$
\begin{aligned}
& \frac{d_{2}}{w_{4}} \leq \frac{a h}{w_{1}}, \\
& d_{1}<\frac{w_{2} x^{*}}{h+x^{* 2}},
\end{aligned}
$$

where

$$
y^{*}=\frac{d_{2}}{w_{4}} ; z^{*}=\frac{1}{w_{3}}\left(\frac{w_{2} x^{*}}{h+x^{* 2}}-d_{1}\right) .
$$

While $x^{*}$ is a positive root of the following equation

$$
b x^{3}-a x^{2}+b h x+\left(w_{1} \frac{d_{2}}{w_{4}}-a h\right)=0 .
$$

According to Descartes's rule of signs, Eq. (6) has either one positive root (given by $E_{3}$ ) and two complex conjugate roots or three positive roots. Straightforward computation using Mathematica software shows that Eq. (6) has only one positive root with two complex conjugate roots.

Now, the local dynamical behavior of the food chain system (1) near the above equilibrium points is investigated. It is observed that the eigenvalues of the variational matrix of system (1) near the trivial equilibrium point $\left(E_{0}\right)$ are determined as

$$
\gamma_{01}=a>0 ; \gamma_{02}=-d_{1}, \gamma_{03}=-d_{2},
$$

while the eigenvalues near the axial equilibrium point $\left(E_{1}\right)$ are given by

$$
\gamma_{11}=-a<0 ; \gamma_{12}=\frac{w_{2} a b}{h b^{2}+a^{2}}-d_{1}, \gamma_{13}=-d_{2} \text {. }
$$

Hence, the trivial equilibrium point is a saddle point, while the axial equilibrium point is locally asymptotically stable provided that the following condition holds

$$
\frac{w_{2} a b}{h b^{2}+a^{2}}<d_{1} \text {. }
$$

Moreover, the eigenvalues of system (1) near the planar equilibrium point $E_{2}$ can be written as:

$$
\gamma_{21}=\frac{T}{2}-\frac{\sqrt{T^{2}-4 D}}{2} ; \gamma_{22}=\frac{T}{2}+\frac{\sqrt{T^{2}-4 D}}{2}, \gamma_{23}=w_{4} \bar{y}-d_{2},
$$

where $T=\bar{x}\left[-b+\frac{2 w_{1} \bar{x} \bar{y}}{\left(h+\bar{x}^{2}\right)^{2}}\right]$ and $D=\frac{w_{1} w_{2}\left(h-\bar{x}^{2}\right) \bar{x} \bar{y}}{\left(h+\bar{x}^{2}\right)^{3}}$. Thus, according to these eigenvalues, if the condition (3a) holds then both the planar equilibrium points are saddle points due to the negativity of $D$ that leads to the existence of positive eigenvalues. However, if the condition (3b) holds, then the unique planar equilibrium point becomes non-hyperbolic point due to the existence of zero eigenvalue and hence the linearization does not give the real stability type.

Now, for the positive equilibrium point, the variational matrix of system (1) around $E_{3}=\left(x^{*}, y^{*}, z^{*}\right)$ is computed as:

where

$$
V\left(x^{*}, y^{*}, z^{*}\right)=\left(v_{i j}\right)_{3 \times 3}
$$

$$
\begin{aligned}
& v_{11}=-b x^{*}+\frac{w_{1} x^{*^{2}} y^{*}}{B^{*^{2}}}, \quad v_{12}=-\frac{w_{1} x^{*}}{B^{*}}, \quad v_{13}=0, \quad v_{21}=\frac{w_{2} y^{*}\left(h-x^{*^{2}}\right)}{B^{*^{2}}}, \\
& v_{22}=\frac{w_{2} x^{*}}{B^{*}}-b-w_{3} z^{*}, \quad v_{23}=-w_{3} y^{*}, \quad v_{31}=0, \quad v_{32}=w_{4} z^{*}, \quad v_{33}=0 .
\end{aligned}
$$

here $B^{*}=h+x^{*^{2}}$. Then the characteristic equation of the variational matrix (11) can be written as

where

$$
\lambda^{3}+K_{1} \lambda^{2}+K_{2} \lambda+K_{3}=0
$$

$$
\begin{aligned}
& K_{1}=b x^{*}-\frac{2 w_{1} x^{*^{2}} y^{*}}{B^{*^{2}}} \\
& K_{2}=\frac{w_{1} w_{2} x^{*} y^{*}\left(h-x^{*^{2}}\right)}{B^{*^{3}}}+w_{3} w_{4} y^{*} z^{*}
\end{aligned}
$$


$K_{3}=\left(b x^{*}-\frac{2 w_{1} x^{*^{2}} y^{*}}{B^{*^{2}}}\right)\left(w_{3} y^{*}\right)\left(w_{4} z^{*}\right)$

while

$K_{1} K_{2}-K_{3}=\left(b x^{*}-\frac{2 w_{1} x^{*^{2}} y^{*}}{B^{*^{2}}}\right)\left(\frac{w_{1} x^{*}}{B^{*}}\right)\left(\frac{w_{2} y^{*}\left(h-x^{*^{2}}\right)}{B^{*^{2}}}\right)$

Clearly, $K_{1}>0, K_{3}>0$ and $K_{1} K_{2}-K_{3}>0$ if and only if the following conditions are fulfilled

$$
x^{*^{2}}<h \quad \frac{2 w_{1} x^{*} y^{*}}{B^{*^{2}}}<b
$$

Therefore, according to the Routh-Hurwitz criterion [22], the locally asymptotically stable $E_{3}=$ $\left(x^{*}, y^{*}, z^{*}\right)$ takes place. However, the region of the global stability of the positive equilibrium point is investigated in the following theorem.

Theorem 1. Assume that $E_{3}=\left(x^{*}, y^{*}, z^{*}\right)$ of system (1) is locally asymptotically stable and the following condition holds

$$
\begin{aligned}
& \frac{w_{1} y^{*}\left(x+x^{*}\right)}{B B^{*}}<b \\
& x y^{*}+x^{*} y<x y+x^{*} y^{*} \\
& \frac{w_{2}}{B}<\frac{w_{1}}{B}+\frac{w_{2}\left(x+x^{*}\right)}{B B^{*}}
\end{aligned}
$$

where $B=\left(h+x^{2}\right)$. Then, the basin of attraction in the Int. $\left(R_{+}^{3}\right)$ of $E_{3}$ satisfies the above conditions. Proof. . Consider the positive definite function

$$
L(x, y, z)=\int_{x^{*}}^{x} \frac{\left(X-x^{*}\right)}{X} d X+\int_{y^{*}}^{y} \frac{\left(Y-y^{*}\right)}{Y} d Y+\frac{w_{3}}{w_{4}} \int_{Z^{*}}^{Z} \frac{\left(Z-z^{*}\right)}{Z} d Z,
$$

Taking the derivative of $L$ with respect to $t$ along the solutions of system (1), we get after some algebraic manipulation that

$$
\frac{d L}{d t}=-\left[b-\frac{w_{1} y^{*}\left(x+x^{*}\right)}{B B^{*}}\right]\left(x-x^{*}\right)^{2}-\left[\frac{w_{1}}{B}-\frac{w_{2}}{B}+\frac{w_{2}\left(x+x^{*}\right)}{B B^{*}}\right]\left(x-x^{*}\right)\left(y-y^{*}\right)
$$

Clearly, under the given conditions that $\frac{d L}{d t}$ is negative semi definite and then by using Lyapunov method, the positive equilibrium point is stable. Moreover, it is easy to verify that, by using LaSalle's invariance principle $\quad, E_{3}$ is the only invariant set for which the solution is approached asymptotically and hence it becomes an attracting point. Therefore, $E_{3}$ is an asymptotically stable point for any initial point that belongs to the region that is satisfying the above conditions.

\section{Numerical exploration}

The global dynamics of system (1) is studied numerically using six order Runge-Kutta method. Many cases of system (1) are obtained for the following set of fixed parameters values

$$
a=0.7, b=0.91, h=0.15, w_{2}=5.95, w_{3}=0.9, w_{4}=0.6, d_{1}=0.0005, d_{2}=0.01
$$

Bifurcation diagram and the typical 3D attractors of system (1) are plotted with their projections and time series in the boundary planes. The fundamental target is to investigate the possibility of chaotic behavior in system (1) by relying upon the controlling parameter and keeping different parameters of (15) fixed.

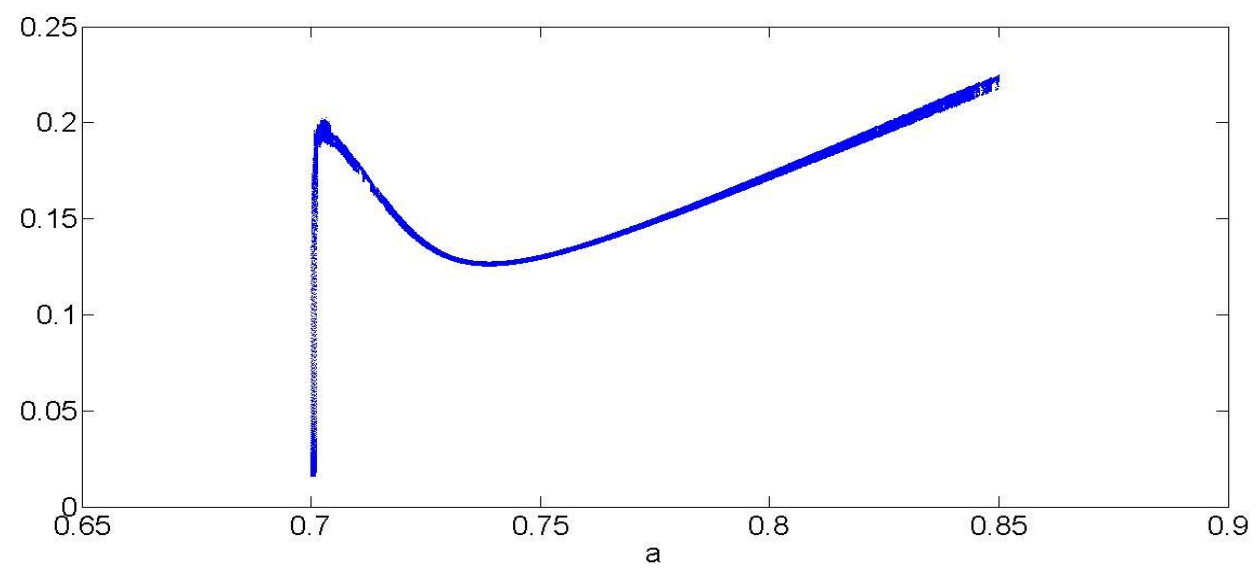

Figure 1- Bifurcation diagram of system (1) for data set (15) that showing the successive maxima of $y$ as a function of $a \in(0.65,0.90)$ and $w_{1}=6.1$. 


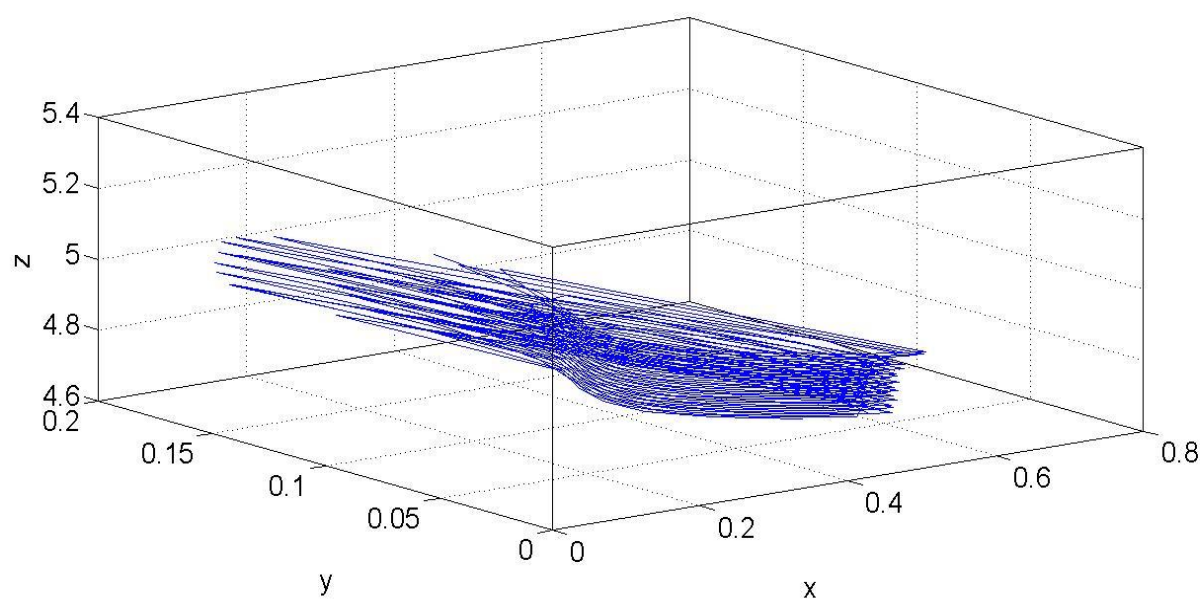

Figure 2- 3D chaotic attractor of system (1) for data set (15) with $w_{1}=6.1$

Now, in the first case when $w_{1}=6.1$ with the data set (15), it is observed that system (1) is chaotic and approaches to periodic attractor, as shown in Figure-2 and Figure-3a-b. In the second case, by decreasing the value of $w_{1}$ to 4.1 , system (1) approaches to a limit cycles attractor, as shown in Figure$4 \mathrm{a}-\mathrm{b}$. The third case deals with keeping on decreasing the values of $w_{1}$ and $d_{2}$ to 0.001 for data (15), where we observe the change in the dynamics of system (1) from limit cycles attractor to stable point, as blotted in Figure-5a-b.
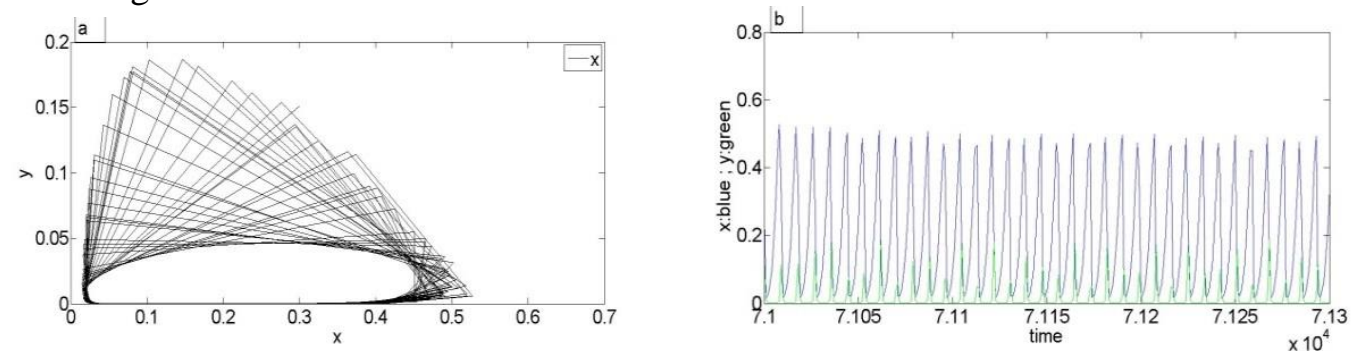

Figure 3- a) 2D projection in the $x y$ - plane of the chaotic attractor given by Figure-2, b) Time series of Figure-3a
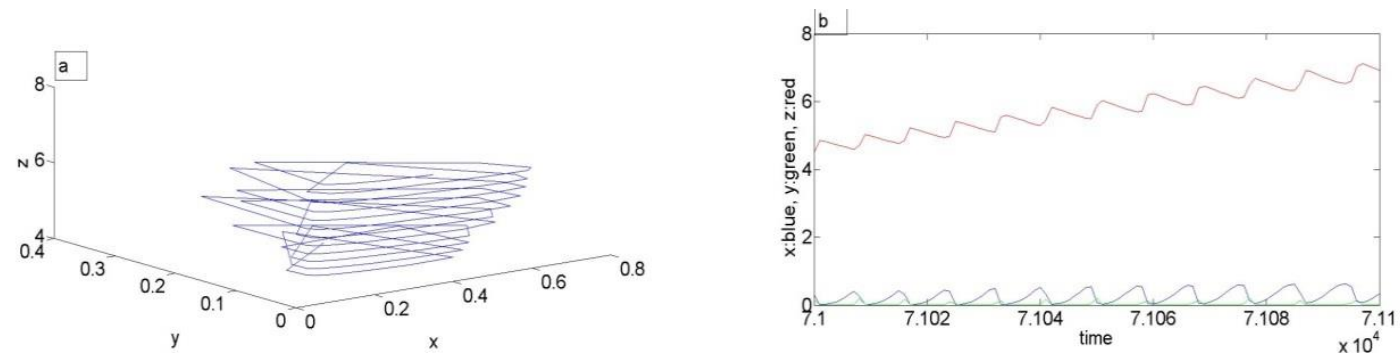

Figure 4- a) 3D of system (1) periodic attractor for data (15) with $w_{1}=4.1$,

b) Time series of Figure-4a. 

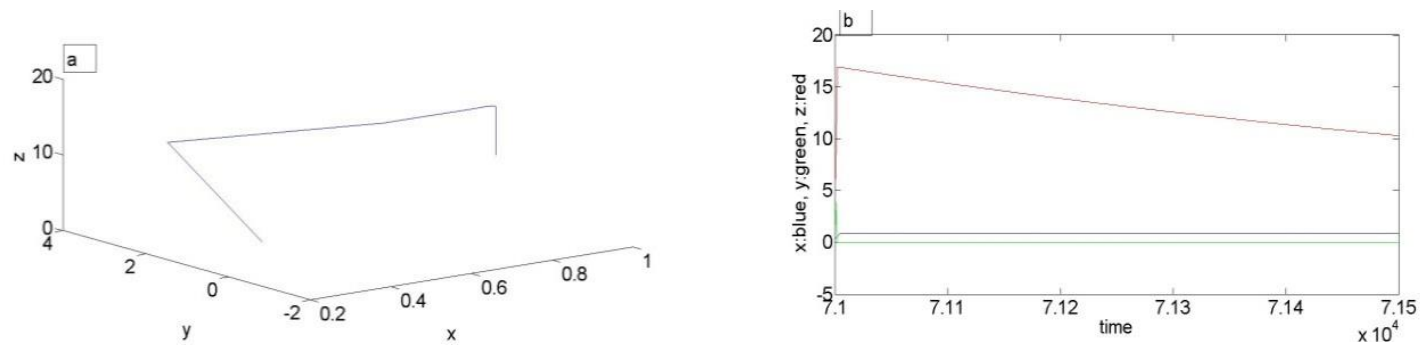

Figure 5- a) 3D of system (1) stable point for data (15) with $w_{1}=d_{2}=0.001$,

b) Time series of Figure-5a

\section{Conclusions}

A food chain system with modified Holling type IV and Lotka-Volterra functional responses is proposed and discussed. The global stability of the system is investigated using Routh-Hurwitz criterion and the second method of Lyapunov. Bifurcation diagram and attractors are blotted to study the behavior of the hybrid continuous time model. The parameter $w_{1}$ is controlling the dynamics of this food chain model. Moreover, replacing Holling type-II functional response in [9] by LotkaVolterra response in system (1) above reduces the effect of nonlinearity and makes the dynamics less chaotic. In fact, the system approaches clearly a periodic behavior.

\section{References}

1. Lotka, A. J. 1925. Elements of Physical Biology, Williams and Wilkins New York.

2. Volterra, V. 1927. Varia zioni e fluttu a zioni del numero d'indidui in speci animali conviventi, Memoria R. Comita to talassografica Italiano, 131: 1-142.

3. Mandel, S. Ray, S. Roy, S. and Jorgensen, S. E. 2006. Order to chaos and vise versa in an aquatic ecosystem, Ecological Model, 197: 498-504.

4. Xu, F. Shu, X. and Cressman, R. 2013. Chaos Control and Chaos Synchronization of Fractional Order Smooth Chua's System, Dynamics of Continuous, Discrete and Impulsive Systems, Series B, 20: 117-134.

5. Petrovskii, S. V. and Malchow, H. 2001. Wave of chaos: New mechanism of pattern formation in spatio-temporal population dynamics, Theoretical Population Biology, 59: 157-174.

6. Naji, R. K. 2012. Global stability and persistence of three species food web involving omnivory, Iraqi Journal of Science, 53(4): 866-876.

7. Datta, J. Jana, D. and Upadhyay, R. K. 2019. Bifurcation and bio-economic analysis of preygeneralist predator with Holling type IV functional response and nonlinear age-selective prey harvesting, Chaos, Solitons and Fractals, 122: 229-235.

8. Ali, S. J. Arifin, N. M. Naji, R. K. Ismail, F and Bachok, N. 2016. Analysis of Ecological Model with Holling Type IV Functional Response, International Journal of Pure and Applied Mathematics, 106(1): 317-331.

9. Ali, S. J. Arifin, N. M. Naji, R. K. Ismail, F and Bachok, N. 2016. Controlling Chaotic Dynamics of a Continuous Ecological Model, International Journal of Pure and Applied Mathematics, 109(2): 177-191.

10. Ali, S. J. Arifin, N. M. Naji, R. K. Ismail, F and Bachok, N. 2019. Global Stability of a Three Species Predator-Prey Food Chain Dynamics, Dynamics of Continuous, Discrete and Impulsive Systems, Series B, 26(4): 39-52.

11. Ali, S. J. 2019. Study of a Predator-Prey Model with Modified Ratio-Dependent and Sokol-Howell Functional Response, SICAPM, 2: 53-57.

12. Guo, L. Song, Z. G. and Xu, J. 2014. Complex dynamics in the Leslie-Gower type of the food chain system with multiple delays, Communications in Nonlinear Science and Numerical Simulation, 19(8): 2850-2865.

13. Chen, G. 1999. Controlling Chaos and Bifurcations in Engineering Systems, Boca Raton, CRC Press.

14. Naji, R. K. and Ibrahim, H. A. 2012. The impact of disease harvesting on the dynamical behavior of prey predator model, Iraqi Journal of Science, 53(1): 130-139. 
15. Panday, P. Pal, N. Samanta, S. and Chattopadhyay, J. 2018. Stability and bifurcation analysis of a three-species food chain model with fear, International Journal of Bifurcation and Chaos, 28(1): 1850009. DOI:10.1142/S0218127418500098

16. Hsu, T. H. 2019. Number and stability of relaxation oscillations for predator-prey system with small death Rates, SIAM, Journal on applied dynamical systems, 18: 33-67.

17. Al-Nassir, S. and Abdullah, F. K. 2017. The dynamics of discrete system with constant rate harvesting, Iraqi Journal of Science, 58(4B): 2177-2187.

18. Beak, H. Lim, Y. and Lim, D. 2011. A Three Species Food Chain System with Two Types of Functional Responses, Abstract and Applied Analysis, 2011: 155-159. DOI:10.1155/2011/934569

19. Upadhyay, R. K. Mishra, S. Dong, Y. and Takeuchi, Y. 2019 Exploring the dynamics of a tritrophic food chain model with multiple gestation periods, Mathematical Biosciences and Engineering, 16(5): 4660-4691.

20. Fayeldi, T. Suryanto, A. and Widodo, A. 2013. Dynamical behaviors of a discrete SIR epidemic model with nonmonotone incidence rate, International Journal of Applied Mathematics and Statistics, 47: 416-423.

21. Liu, X. and Huang, Q. 2018. The dynamics of a harvested predator-prey with Holling type IV functional response, Biosystems, 169: 26-39.

22. Allen, L. JS. 2007 Introduction to mathematical biology, Pearson-Prentice Hall, New-Jersey. 